\title{
Correction to: Rapid Acquisition of High-Quality SARS-CoV-2 Genome via Amplicon-Oxford Nanopore Sequencing
}

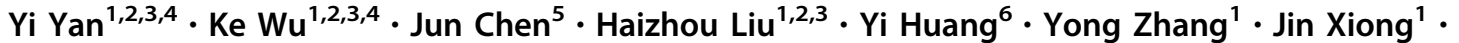 \\ Weipeng Quan ${ }^{7} \cdot \mathrm{Xin} \mathrm{Wu}^{8} \cdot \mathrm{Yu} \mathrm{Liang}^{9} \cdot \mathrm{Kunlun} \mathrm{He}^{10,11} \cdot$ Zhilong Jia $^{10,11} \cdot$ Depeng Wang $^{8} \cdot \mathrm{Di} \mathrm{Liu}^{1,2,3,4,12}$ (1) \\ Hongping Wei ${ }^{1} \cdot$ : Jianjun Chen ${ }^{1,2}$ (i)
}

Published online: 17 November 2021

(C) Wuhan Institute of Virology, CAS 2021

\section{Correction to: \\ Virologica Sinica (2021) 36:901-912 https://doi.org/10.1007/s12250-021-00378-8}

The original version of article contains errors in Figure 4, Supplementary Figure S2, Supplementary Table S2, and the corresponding description in part 3 of "Results" section. The corrected contents are shown below:

1. Figure 4

2. Supplementary Figure S2

3. Supplementary Table $\mathbf{S} 2$

4. Part 3 of the "Results" section:

Yi Yan, Ke Wu, and Jun Chen have contributed equally to this work.

The original article can be found online at https://doi.org/10.1007/ s12250-021-00378-8.

\section{Jianjun Chen \\ chenjj@wh.iov.cn \\ $\triangle$ Hongping Wei \\ hpwei@wh.iov.cn \\ liud@wh.iov.cn}

$凶$ Di Liu

1 CAS Key Laboratory of Special Pathogens and Biosafety, Wuhan Institute of Virology, Center for Biosafety MegaScience, Chinese Academy of Sciences, Wuhan 430071, China

2 National Virus Resource Center, Wuhan Institute of Virology, Chinese Academy of Sciences, Wuhan 430071, China

3 Computational Virology Group, Center for Bacteria and Viruses Resources and Bioinformation, Wuhan Institute of Virology, Chinese Academy of Sciences, Wuhan 430071, China

4 University of Chinese Academy of Sciences, Beijing 101409, China
Subtitle: High-quality Genome Revealed Genetic Variations of SARS-CoV-2

By using bcftools mpileup and Medaka for SNP calling and a published script (margin_cons.py; Quick et al. 2017) for consensus generation, 38 nearly full-length (on average about $99.61 \%$ ) SARS-CoV-2 genomes with high-quality, and 4 shorter genomes (from 93.92 to $97.68 \%$ ) with gaps were obtained. All these genomes have been deposited in NGDC (accessions: GWHALPE01000000-GWHALPT010 00000 and GWHALRI01000000-GWHALSH01000000) and GISAID database (accessions: EPI_ISL_493149EPI_ISL_493190). Compared to an early virus isolate genome IVDC-HB-01 (GISAID accession number: EPI_ISL_402119), 44 SNP sites were discovered in the 38 nearly full-length genomes, with 21 synonymous mutation sites and 23 nonsynonymous ones (Fig. 4, Supplementary Table S2). For each virus genome, there were 0 to 5 SNPs compared to IVDC-HB-01. For all SNP sites, only one mutated nucleotide existed. Through comparison with all deposited genome sequences in GISAID database, as of submitted date of March 1, 2020, 7 of the SNPs were

5 Wuhan Pulmonary Hospital, Wuhan Tuberculosis Prevention and Treatment Institute, Wuhan 430030, China

6 National Biosafety Laboratory, Chinese Academy of Sciences, Wuhan 430071, China

7 GrandOmics Biosciences, Wuhan 430000, China

8 GrandOmics Biosciences, Beijing 102200, China

9 GrandOmics Diagnostics, Wuhan 430000, China

10 Key Laboratory of Biomedical Engineering and Translational Medicine, Ministry of Industry and Information Technology, Chinese PLA General Hospital, Beijing 100039, China

11 Beijing Key Laboratory for Precision Medicine of Chronic Heart Failure, Chinese PLA General Hospital, Beijing 100039, China

12 First Affiliated Hospital of Xinjiang Medical University, Urumqi 830054, China 


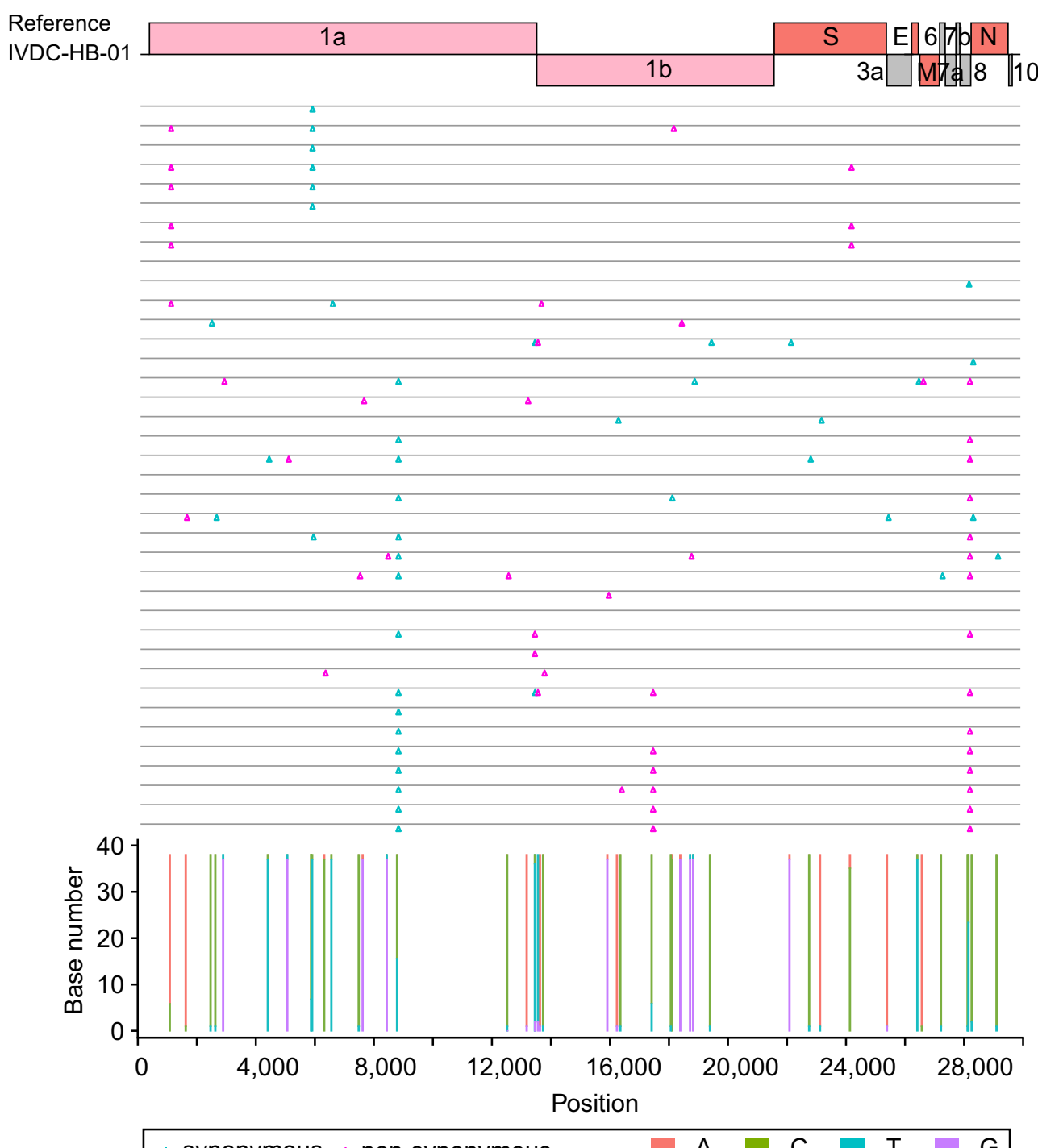

Fig. 4 Nucleotide and amino acid variations in 38 sample genomes. The top shows the open reading frames (ORFs) position of the reference genome, and the middle displays the amino acid variations on the genome of each sample. The blue triangle represents synonymous mutation, the red triangle represents non-synonymous

appeared in previous released genome sequences (before the date of the given sample collected) as well as observed in the following virus genomes, 37 SNPs were firstly mutation. The bottom shows the nucleotide composition at the mutation sites identified in the 38 samples. Different colors represent different bases, the bar in red is adenine (A), the bar in green is cytosine $(\mathrm{C})$, the bar in blue is thymine $(\mathrm{T})$, and the bar in purple is guanine $(\mathrm{G})$

discovered at the date of sample collection (Supplementary Fig. S2, Supplementary Table S2). 